\title{
COMPOSITION OF OPERATOR IDEALS
}

\author{
MIKAEL LINDSTRÖM and GEORG SCHLÜCHTERMANN
}

\section{Introduction}

In $[13,14]$ E. Saksman and H. Tylli and G. Racher in [10] have been studying weak compactness for continuous linear composition operators $S_{R B}: \mathscr{L}\left(F_{1}, E\right) \rightarrow \mathscr{L}\left(E_{1}, F\right)$ defined by $T \mapsto R \circ T \circ B$. Here $E, F, E_{1}, F_{1}$ are Banach spaces and we suppose that $R \in \mathscr{L}(E, F)$ and $B \in \mathscr{L}\left(E_{1}, F_{1}\right)$. If $E^{*}$ or $F_{1}$ has the Dunford-Pettis property, then G. Racher [10] has shown that $S_{R B}$ is weakly compact if and only if $R$ and $B$ are weakly compact operators. In [14] E. Saksman and H. Tylli point out that the exact condition for $S_{R B}$ to be weakly compact is in general quite complicated and to a large extent unknown.

In this paper we try to unify these results by considering abstract Banach and operator ideals (see 2.1-2.3 below). Beside the results on weak compactness of the composed operator $S_{R B}$, we investigate other properties of $S_{R B}$, namely when the operator is Asplund or Rosenthal. For this purpose we study the so-called fine line betwen Asplund and conditionally weakly compact sets in the space $L_{\infty}^{c}(\mu, F)$ of measurable functions with $\mu$-essentially relatively compact range which may be of independent interest and which extends results of H. Collins, W. Ruess and C. Stegall. Section 3 answers the question that the given criteria in part two for the composed operator to be weakly compact, Asplund or Rosenthal are in certain cases necessary.

For Banach spaces $E, F$ let $\mathscr{F}(E, F), \mathscr{K}(E, F), \mathscr{W}(E, F), \mathscr{R}(E, F)$, $\mathscr{D}_{\infty}(E, F)$ denote the spaces of all finite rank, compact, weakly compact, Rosenthal and Asplund operators from $E$ into $F$ respectively. Let us recall that $T$ is called compact, weakly compact, Rosenthal, Asplund, if it maps the closed unit ball of $E$ onto a relatively compact, a relatively weakly compact, a conditionally weakly compact, an Asplund set in $F$. A subset $K$ of $E$ is called an Asplund set, if $\left(\left(\overline{\operatorname{span}} K_{0}\right)^{*}, p_{K_{0}}\right)$ is separable for all countable $K_{0} \subset K$, where $p_{K_{0}}\left(x^{*}\right):=\sup _{x \in K_{0}}\left|<x, x^{*}>\right|, x^{*} \in\left(\overline{\operatorname{span}} K_{0}\right)^{*}$ (see [1], pp.

Received October 25. 1996. 
116, and [11] for the definition and further results). Recall also that a Banach space $E$ is an Asplund space if the closed unit ball $B_{E}$ of $E$ is an Asplund set. That $E$ is an Asplund space if and only if $E^{*}$ has the Radon-Nikodym property was proved by C. Stegall [16]. Furthermore, $T \in \mathscr{L}(E, F)$ is called a Dieudonné operator, if every weak Cauchy sequence in $E$ is mapped into a weakly convergent sequence in $F$. The ideal of Dieudonné operators is closed. The celebrated factorization technique of Davis et al. is known to imply that every weakly compact, Rosenthal, Asplund operator factors through a reflexive space, a space not containing a copy of $\ell_{1}$, an Asplund space respectively. We denote by $B_{E}$ the closed unit ball of a Banach space E.

\section{Main results}

We start with an elementary general result. In the case $\mathscr{A}$ is the space of all weakly compact operators and $(\mathscr{B}, \beta)$ is the space of all continuos linear operators with the operator norm this result was proved by Saksman and Tylli ([13], Prop. 2.1). For the basic properties of Banach and operator ideals we refer the reader to $[4,9]$.

Proposition 2.1. Let $E, E_{1}, F, F_{1}$ be Banach spaces and let $R \in \mathscr{L}(E, F)$ and $B \in \mathscr{L}\left(E_{1}, F_{1}\right)$ be non-zero operators. Let $\mathscr{A}$ be an operator ideal, $(\mathscr{B}, \beta)$ a Banach ideal and suppose that the continuous linear operator $S_{R B}: \mathscr{B}\left(F_{1}, E\right) \rightarrow$ $\mathscr{B}\left(E_{1}, F\right), S_{R B}(T):=R \circ T \circ B$, belongs to $\mathscr{A}$. Then $R \in \mathscr{A}(E, F)$ and $B \in \mathscr{A}^{\text {dual }}\left(E_{1}, F_{1}\right)$.

Proof. First we show that $R \in \mathscr{A}(E, F)$. Pick $x_{0}^{*} \in F_{1}^{*}$ and $z_{0} \in E_{1}$ with $x_{0}^{*}\left(B\left(z_{0}\right)\right)=1$. Then we define $s_{1} \in \mathscr{L}\left(E, \mathscr{B}\left(F_{1}, E\right)\right)$ by $s_{1}(x)=x_{0}^{*}(\cdot) x$ and $s_{2} \in \mathscr{L}\left(\mathscr{B}\left(E_{1}, F\right), F\right)$ by $s_{2}(T)=T\left(z_{0}\right)$. Now, for all $x \in E$, we have that $\left(s_{2} \circ S_{R B} \circ s_{1}\right)(x)=R(x)$. Thus $R \in \mathscr{A}(E, F)$. To prove that $B \in \mathscr{A}^{\text {dual }}$ $\left(E_{1}, F_{1}\right)$ take $x_{0} \in E$ and $y_{0}^{*} \in F^{*}$ with $y_{0}^{*}\left(R\left(x_{0}\right)\right)=1$. Now we define $s_{1} \in \mathscr{L}\left(F_{1}^{*}, \mathscr{B}\left(F_{1}, E\right)\right) \quad$ by $\quad s_{1}\left(y^{*}\right)=y^{*}(\cdot) x_{0} \quad$ and $\quad s_{2} \in \mathscr{L}\left(\mathscr{B}\left(E_{1}, F\right), E_{1}^{*}\right) \quad$ by $s_{2}(T)=y_{0}^{*} \circ T$. Then $\left(s_{2} \circ S_{R B} \circ s_{1}\right)\left(y^{*}\right)=B^{*}\left(y^{*}\right)$ for all $y^{*} \in F_{1}^{*}$. Thus $B \in \mathscr{A}^{\text {dual }}\left(E_{1}, F_{1}\right)$.

The next two propositions were inspired by results due to Diestel and Faires [3]. As we shall see, these two results cover some of the recent results by Racher [10], and Saksman and Tylli $[13,14]$.

Proposition 2.2. Let $\mathscr{A}$ be a closed operator ideal and let $(\mathscr{B}, \beta)$ be a $\mathrm{Ba}$ nach ideal. Define, for any pair of Banach spaces $E, F$, the class $\mathscr{A}_{\mathrm{left}}^{\mathrm{comp}}(E, F)$ of all operators $R \in \mathscr{L}(E, F)$ such that for any pair of Banach spaces $E_{1}, F_{1}$ and any operator $B \in \mathscr{A}^{\text {dual }}\left(E_{1}, F_{1}\right)$ the map $S_{R}: \mathscr{B}\left(F_{1}, E\right) \longrightarrow \mathscr{B}\left(E_{1}, F\right)$, de- 
fined by $S_{R}(T):=R \circ T \circ B$, belongs to $\mathscr{A}$. Then $\mathscr{A}_{\text {left }}^{\text {comp }}$ is a closed operator ideal contained in $\mathscr{A}$. If $\mathscr{A}$ and $\mathscr{B}$ are injective, then $\mathscr{A}_{\text {left }}^{\text {comp }}$ is also injective.

Proof. Let $R_{1}, R_{2} \in \mathscr{A}_{\text {left }}^{\text {comp }}(E, F)$. Then by definition $S_{R_{1}}, S_{R_{2}} \in \mathscr{A}$ and therefore $S_{R_{1}+R_{2}}=S_{R_{1}}+S_{R_{2}}$ is in $\mathscr{A}$ from which follows that $R_{1}+R_{2} \in$ $\mathscr{A}_{\text {left }}^{\text {comp }}(E, F)$. Further, let $W, Z$ be Banach spaces and let $A \in \mathscr{L}(W, E)$, $R \in \mathscr{A}_{\text {left }}^{\text {comp }}(E, F)$ and $C \in \mathscr{L}(F, Z)$. The map $S_{C \circ R \circ A}$ can be written as $s_{2} \circ S_{R} \circ s_{1}, \quad$ where $\quad s_{1}: \mathscr{B}\left(F_{1}, W\right) \rightarrow \mathscr{B}\left(F_{1}, E\right), \quad s_{1}(T)=A \circ T, \quad$ and $\quad s_{2}$ : $\mathscr{B}\left(E_{1}, F\right) \rightarrow \mathscr{B}\left(E_{1}, Z\right), s_{2}(T)=C \circ T$. Therefore $C \circ R \circ A \in \mathscr{A}_{\text {left }}^{\text {comp }}(W, Z)$. Next, let $R \in \mathscr{L}(E, F)$ with $R(x)=x_{0}^{*}(x) y_{0}$ for some $x_{0}^{*} \in E^{*}$ and some $y_{0} \in F$. If $E_{1}, \quad F_{1}$ are Banach spaces and $B \in \mathscr{A}^{\text {dual }}\left(E_{1}, F_{1}\right)$, then $S_{R}: \mathscr{B}\left(F_{1}, E\right) \rightarrow \mathscr{B}\left(E_{1}, F\right)$ can be written as $S_{R}=s_{2} \circ B^{*} \circ s_{1}$, where $s_{1}: \mathscr{B}\left(F_{1}, E\right) \rightarrow F_{1}^{*}, \quad s_{1}(T)=x_{0}^{*} \circ T, \quad$ and $\quad s_{2}: E_{1}^{*} \rightarrow \mathscr{B}\left(E_{1}, F\right), \quad s_{2}\left(x^{*}\right) x=$ $x^{*}(x) y_{0}$. Thus $S_{R} \in \mathscr{A}$ and hence $R \in \mathscr{A}_{\text {left }}^{\text {comp }}$. Finally, the inequality

$$
\beta\left(R \circ T \circ B-R_{n} \circ T \circ B\right) \leq\left\|R-R_{n}\right\| \cdot \beta(T) \cdot\|B\|,
$$

shows that $\mathscr{A}_{\text {left }}^{\text {comp }}(E, F)$ is a closed operator ideal.

Let $R \in \mathscr{A}_{\text {left }}^{\text {comp }}(E, F)$. Choose $E_{1}$ and $F_{1}$ to be the scalar field and $B$ to be the identity. Then $R=s_{2} \circ S_{R} \circ s_{1}$, where $s_{1}: E \rightarrow \mathscr{B}(K, E), s_{1}(x) \lambda=\lambda x$ and $s_{2}: \mathscr{B}(K, F) \rightarrow F, s_{2}(u)=u(1)$. Thus $\mathscr{A}_{\text {left }}^{\text {comp }} \subset \mathscr{A}$.

Let $R \in \mathscr{L}(E, F)$ be such that $J_{F} \circ R \in \mathscr{A}_{\text {left }}^{\text {comp }}\left(E, l_{\infty}\left(B_{F^{*}}\right)\right)$, where $J_{F}: F \rightarrow l_{\infty}\left(B_{F^{*}}\right)$ is the canonical injection. Then by definition the map $J_{F} \circ S_{R}=S_{J_{F} \circ R}: \mathscr{B}\left(F_{1}, E\right) \rightarrow \mathscr{B}\left(E_{1}, l_{\infty}\left(B_{F^{*}}\right)\right)$ belongs to $\mathscr{A}$. Now, the injectivity of $\mathscr{A}$ gives that $S_{R}: \mathscr{B}\left(F_{1}, E\right) \rightarrow \mathscr{B}^{i n j}\left(E_{1}, F\right)$ belongs to $\mathscr{A}$ and hence, by the injectivity of $\mathscr{B}, R \in \mathscr{A}_{\text {left }}^{\text {comp }}$. Thus we have shown the injectivity of $\mathscr{A}_{\text {left }}^{\text {comp }}$.

Proposition 2.3. Let $\mathscr{A}$ be a closed operator ideal and let $(\mathscr{B}, \beta)$ be a $B a$ nach ideal. Define, for any pair of Banach spaces $E, F$, the class $\mathscr{A}_{\text {right }}^{\text {comp }}(E, F)$ of all operators $R \in \mathscr{L}(E, F)$ such that for any pair of Banach spaces $E_{1}, F_{1}$ and any operator $B \in \mathscr{A}\left(E_{1}, F_{1}\right)$ the map $S_{R}: \mathscr{B}\left(F, E_{1}\right) \longrightarrow \mathscr{B}\left(E, F_{1}\right)$, defined by $S_{R}(T):=B \circ T \circ R$, belongs to $\mathscr{A}$. Then $\mathscr{A}_{\text {right }}^{\text {comp }}$ is closed operator ideal contained in $\mathscr{A}^{\text {dual }}$. If $\mathscr{A}$ is injective and $\mathscr{B}$ is symmetric, injective and $\beta\left(T^{*}\right) \leq \beta(T)$ for all $T \in \mathscr{B}$, then $\mathscr{A}_{\text {right }}^{\text {comp }}$ is surjective.

Proof. The proof is very similar to the proof of Proposition 2.2, so we shall be brief. Let $W, Z$ be Banach spaces and let $A \in \mathscr{L}(W, E)$, $R \in \mathscr{A}_{\text {right }}^{\text {comp }}(E, F)$ and $C \in \mathscr{L}(F, Z)$. The map $S_{C \circ R \circ A}$ can be written as $s_{2} \circ S_{R} \circ s_{1}, \quad$ where $s_{1}: \mathscr{B}\left(Z, E_{1}\right) \rightarrow \mathscr{B}\left(F, E_{1}\right), \quad s_{1}(T)=T \circ C \quad$ and $\quad s_{2}:$ $\mathscr{B}\left(E, F_{1}\right) \rightarrow \mathscr{B}\left(W, F_{1}\right), s_{2}(T)=T \circ A$. Therefore $C \circ R \circ A \in \mathscr{A}_{\mathrm{right}}^{\text {comp }}(W, Z)$. Next, let $R \in \mathscr{L}(E, F)$ with $R(x)=x_{0}^{*}(x) y_{0}$ for some $x_{0}^{*} \in E^{*}$ and some $y_{0} \in F$. If $E_{1}, \quad F_{1}$ are Banach spaces and $B \in \mathscr{A}\left(E_{1}, F_{1}\right)$, then $S_{R}: \mathscr{B}\left(F, E_{1}\right) \rightarrow \mathscr{B}\left(E, F_{1}\right) \quad$ can be written as $S_{R}=s_{2} \circ B \circ s_{1}$, where 
$s_{1}: \mathscr{B}\left(F, E_{1}\right) \rightarrow E_{1}, s_{1}(T)=T\left(y_{0}\right)$, and $s_{2}: F_{1} \rightarrow \mathscr{B}\left(E, F_{1}\right), s_{2}(y) x=x_{0}^{*}(x) y$. Thus $S_{R} \in \mathscr{A}$ and hence $R \in \mathscr{A}_{\text {right }}^{\text {comp }}$.

It is easy to see that $\mathscr{A}_{\text {right }}^{\text {comp }}(E, F)$ is a closed operator ideal and that $\mathscr{A}_{\text {right }}^{\text {comp }} \subset \mathscr{A}^{\text {dual }}$.

Let $R \in \mathscr{L}(E, F)$ be such that $R \circ Q_{E} \in \mathscr{A}_{\text {right }}^{\text {comp }}\left(\ell_{1}\left(B_{E}\right), F\right)$, where $Q_{E}: \ell_{1}\left(B_{E}\right) \rightarrow E$ is the canonical surjection. Suppose that $B \in \mathscr{A}\left(E_{1}, F_{1}\right)$. Then by definition the map $S_{R \circ Q_{E}}: \mathscr{B}\left(F, E_{1}\right) \rightarrow \mathscr{B}\left(\ell_{1}\left(B_{E}\right), F_{1}\right)$ belongs to $\mathscr{A}$. Thus, $s_{1} \circ S_{R \circ Q} \in \mathscr{A}$, where $s_{1}: \mathscr{B}\left(\ell_{1}\left(B_{E}\right), F_{1}\right) \rightarrow \mathscr{B}\left(F_{1}^{*}, l_{\infty}\left(B_{E^{*}}\right)\right), T \mapsto T^{*}$, is well-defined, since $\mathscr{B}$ is symmetric and it is also continuous, since $\beta\left(s_{1}(T)\right) \leq \beta(T)$ for all $T \in \mathscr{B}\left(\ell_{1}\left(B_{E}\right), F_{1}\right)$. We have $s_{1} \circ S_{R \circ Q}=J_{E^{*}} \circ S_{1} \circ S_{R}$, where $J_{E^{*}}:=\left(Q_{E}\right)^{*}$. The injectivity of $\mathscr{A}$ gives that $s_{1} \circ S_{R}: \mathscr{B}\left(F, E_{1}\right) \rightarrow$ $\mathscr{B}^{\text {inj }}\left(F_{1}^{*}, E^{*}\right)$ belongs to $\mathscr{A}$. Define $s_{2}: \mathscr{B}\left(F_{1}^{*}, E^{*}\right) \rightarrow \mathscr{B}\left(E^{* *}, F_{1}^{* *}\right), T \mapsto T^{*}$, and $s_{3}: \mathscr{B}\left(E^{* *}, F_{1}^{* *}\right) \rightarrow \mathscr{B}\left(E, F_{1}^{* *}\right), T \mapsto T \circ J_{E}$, where $J_{E}: E \rightarrow E^{* *}$ is the canonical map, which are well-defined and continuous by the assumption. Since $\mathscr{B}$ is injective, $J_{F_{1}} \circ S_{R}=s_{3} \circ s_{2} \circ S_{1} \circ S_{R} \in \mathscr{A}$ and hence $\mathscr{A}_{\text {right }}^{\text {comp }}$ is surjective.

Corollary 2.4. Let $\mathscr{A}$ be a closed operator ideal, such that $\mathscr{A}=\mathscr{A}^{\text {dual }}$. Let $(\mathscr{B}, \beta)$ be a Banach ideal, $E, F, E_{1}, F_{1}$ be Banach spaces, $R \in \mathscr{A}(E, F)$ and $B \in \mathscr{A}\left(E_{1}, F_{1}\right)$. If $R$ or $B$ is an approximable operator (i.e. operators which are the norm limit of finite rank operators), then the map $S_{R B}$ : $\mathscr{B}\left(F_{1}, E\right) \rightarrow \mathscr{B}\left(E_{1}, F\right), S_{R B}(T):=R \circ T \circ B$, belongs to $\mathscr{A}$.

Proof. Since the ideal of approximable operators is the smallest closed operator ideal, propositions 2.2 and 2.3 give the statement.

Corollary 2.5. Let $(\mathscr{B}, \beta)$ be a Banach ideal. Let $E, F, E_{1}, F_{1}$ be Banach spaces, such that at least one of the spaces $E^{*}, E_{1}^{*}, F, F_{1}$ has the approximation property. If $R \in \mathscr{L}(E, F)$ and $B \in \mathscr{L}\left(E_{1}, F_{1}\right)$ are non-zero operators, then $R$ and $B$ are compact if and only if $S_{R B}: \mathscr{B}\left(F_{1}, E\right) \rightarrow \mathscr{B}\left(E_{1}, F\right)$, $S_{R B}(T):=R \circ T \circ B$, is a compact operator.

Proof. We apply Proposition 2.1, Corollary 2.4 and the fact that $R$ or $B$ is an approximable operator.

Corollary 2.6. Let $\mathscr{A}$ be an injective closed operator ideal, such that $\mathscr{A}=\mathscr{A}^{\text {dual }}$. Let $(\mathscr{B}, \beta)$ be a Banach ideal, $E, F, E_{1}, F_{1}$ be Banach spaces, $R \in \mathscr{A}(E, F)$ and $B \in \mathscr{A}\left(E_{1}, F_{1}\right)$. The map $S_{R B}: \mathscr{B}\left(F_{1}, E\right) \rightarrow \mathscr{B}\left(E_{1}, F\right)$, $S_{R B}(T):=R \circ T \circ B$, belongs to $\mathscr{A}$

a) if $\mathscr{B}$ is injective and $R$ is compact or

b) if $\mathscr{B}$ is injective, symmetric with $\beta(T) \leq \beta\left(T^{*}\right)$ for all $T \in \mathscr{B}$ and $B$ is compact.

Proof. The closed ideal of compact operators is the smallest closed in- 
jective ideal and also the smallest closed surjective ideal. Now we apply propositions 2.2 and 2.3 to a) and b), respectively.

In the case $\mathscr{B}=\mathscr{L}$ with the operator norm the following result is an extension of Schauder's theorem and is due to K. Vala [17].

Corollary 2.7. Let $(\mathscr{B}, \beta)$ be an injective Banach ideal. Let $E, F, E_{1}, F_{1}$ be Banach spaces. If $R \in \mathscr{L}(E, F)$ and $B \in \mathscr{L}\left(E_{1}, F_{1}\right)$ are non-zero operators, then $R$ and $B$ are compact if and only if $S_{R B}: \mathscr{B}\left(F_{1}, E\right) \rightarrow$ $\mathscr{B}\left(E_{1}, F\right), S_{R B}(T):=R \circ T \circ B$, is a compact operator.

If we apply the above Corollary 2.6 , when $\mathscr{A}$ is the ideal $\mathscr{W}$ of all weakly compact operators and $\mathscr{B}=\mathscr{L}$ with the operator norm, then we obtain Theorem 2.9 in [13]. In [14, Theorem 2.(i)] Saksman and Tylli give another proof of this result. Racher obtained it as well as many other results as immediate consequences of his Main Lemma [10].

In the following we shall prove a similar result, which also contains the Main Lemma of Racher. Our approach will be based on an careful investigation of the space $L_{\infty}^{c}(\mu, F)$ of $\mu$-measurable, Banach space valued functions with $\mu$-essential relatively compact range, where $(\Omega, \Sigma, \mu)$ is a positive and finite measure space. The proof will use results presented in [15]. For this purpose we consider the fine line between conditionally weakly compact and Asplund sets in $L_{\infty}^{c}(\mu, F)$-spaces. In the following we describe the general situation: Let $K:=\left\{h_{n} ; n \in \mathrm{N}\right\} \subset L_{\infty}^{c}(\mu, F)$ be bounded. We select a sequence of representatives of the equivalence classes $\left(h_{n}\right)$ and call this sequence again $\left(h_{n}\right)$. We may assume that $\left\|h_{n}(\omega)\right\| \leq\left\|h_{n}\right\|_{\infty}$ for all $\omega \in \Omega$. Then according to $\left[15\right.$, p.276,2.8] we consider $\mathscr{C}_{K}:=\left\{f \in(\overline{\operatorname{span}} K)^{*}\right.$; $\exists\left(\omega_{k}, y_{k}^{*}\right) \in \Omega^{N} \times B_{F^{*}}^{N}, \lim _{k \rightarrow \infty}\left\langle h_{n}\left(\omega_{k}\right), y_{k}^{*}\right\rangle=: f\left(h_{n}\right)$ exists for all $\left.n \in \mathrm{N}\right\}, \quad$ a subset of the dual unit ball of $L_{\infty}^{c}(\mu, F)$. According to [15, p.279] with the help of this $w^{*}$-compact set and a regular Borel-measure $\nu$, which is measure isomorphic to the Lebesgue measure on $[0,1]$, one can determine, whether the set $K$ is Asplund or conditionally weakly compact. In the following lemma this general description of the functionals will be reduced to an expression, which is easier to handle.

LemMa 2.8. Let $(\Omega, \Sigma, \mu)$ be a finite and positive measure space and let $F$ be a separable Banach space. Let $\left(h_{n}\right) \subset L_{\infty}^{c}(\mu, F)$ be bounded. Then there exists a set $N \subset \Omega$ of measure zero, such that

$$
\begin{gathered}
\mathscr{C}_{K}=\left\{f \in(\overline{\operatorname{span}} K)^{*} ; \exists\left(\omega_{k}\right) \subset \Omega \backslash N \exists y^{*} \in B_{F^{*}}\right. \text { such that } \\
\left.\lim _{k \rightarrow \infty} h_{n}\left(\omega_{k}\right) \text { exists in norm and } f\left(h_{n}\right)=\lim _{k \rightarrow \infty}\left\langle h_{n}\left(\omega_{k}\right), y^{*}\right\rangle\right\} .
\end{gathered}
$$

Proof. Let $f \in \mathscr{C}_{K}$ and $\left(\left(\omega_{k}\right),\left(y_{k}^{*}\right)\right)$ be such that $f\left(h_{n}\right)=\lim _{k \rightarrow \infty}$ 
$\left\langle h_{n}\left(\omega_{k}\right), y_{k}^{*}\right\rangle$. Since all $h_{n}$ have $\mu$-essential relatively compact range, there is a set $N$ of measure zero, such that $h_{n}(\Omega \backslash N)$ is relatively compact for $n \in \mathrm{N}$. Hence, there exists a subsequence $\left(\omega_{k_{j}}\right) \subset \Omega \backslash N$, such that $\left(h_{n}\left(\omega_{k_{j}}\right)\right)$ converges in norm for all $n \in \mathrm{N}$ and $y_{k_{j}}^{*} \rightarrow y^{*} \in B_{F^{*}}$ in the $w^{*}$-topology. Thus

$$
f\left(h_{n}\right)=\lim _{k \rightarrow \infty}\left\langle h_{n}\left(\omega_{k}\right), y_{k}^{*}\right\rangle=\lim _{j \rightarrow \infty}\left\langle h_{n}\left(\omega_{k_{j}}\right), y^{*}\right\rangle .
$$

We will now consider for our investigation of the fine line a countable bounded subset $K$, such that the $\overline{\operatorname{span}}\left\{y^{*} \circ h ; y^{*} \in B_{F^{*}}, h \in K\right\} \subset L_{\infty}(\mu)$ has separable dual. This is necessary, since even in the scalar case, $\ell_{1} \subset C([0,1])$ isometrically.

Let $\tilde{K}:=\left\{y^{*} \circ h ; y^{*} \in B_{F^{*}}, h \in K\right\} \subset L_{\infty}(\mu)$. Then the corresponding $w^{*}$ compact set $\mathscr{C}_{\tilde{K}} \subset L_{\infty}(\mu)$ is norm separable according to the previous assumption. Hence, there is a dense sequence $\left(F_{j}\right) \subset \mathscr{C}_{\tilde{K}}$ with corresponding representation

$$
F_{j}(h)=\lim _{k \rightarrow \infty} h\left(\omega_{k}^{j}\right),\left(\omega_{k}^{j}\right) \subset \Omega \text { suitable, } h \in \tilde{K} .
$$

The definition of $\tilde{K}$ and the description of $\mathscr{C}_{K}$ in 2.8 yield that the corresponding sequences $\left(\omega_{k}\right) \subset \Omega$ may be the same for $\mathscr{C}_{K}$ and $\mathscr{C}_{\tilde{K}}$, by going to subsequences if necessary. For $j \in \mathrm{N}, y^{*} \in B_{F^{*}}$ define $f_{j, y^{*}} \in \mathscr{C}_{K}$ by $f_{j, y^{*}}\left(h_{n}\right):=F_{j}\left(y^{*} \circ h_{n}\right)$.

If $\delta>0, j \in \mathrm{N}$ then we define $C_{\delta_{j} j}:=\left\{f \in \mathscr{C}_{K} ; \exists y^{*} \in B_{F^{*}}\right.$ such that $\left.\left\|f-f_{j, y^{*}}\right\| \leq \delta\right\}$. Since $\mathscr{C}_{K}$ is $w^{*}$-compact according to [15, 3.4 Lemma,p.279] and since the norm is $w^{*}$-lower semicontinuous the set $C_{\delta, j}$ is $w^{*}$-compact. The density of $\left(F_{j}\right) \subset \mathscr{C}_{\tilde{K}}$ and the description of $\mathscr{C}_{K}$ gives $\mathscr{C}_{K}=\bigcup_{j \in \mathrm{N}} C_{\delta, j}$.

TheOREM 2.9. Let $(\Omega, \Sigma, \mu)$ be a positive and finite measure space, let $F$ be a real Banach space. Let $K \subset L_{\infty}^{c}(\mu, F)$ be bounded and countable, such that $\overline{\operatorname{span}}\left\{y^{*} \circ h ; y^{*} \in B_{F^{*}}, h \in K\right\} \subset L_{\infty}(\mu)$ has separable dual. Then

a) $K$ is conditionally weakly compact, if $\ell_{1} \not \subset F$.

b) $K$ is an Asplund set, provided $F$ is an Asplund space.

Proof. We split the proof into three steps and assume w.l.o.g. that $K \subset B_{L_{\infty}^{c}(\mu, F)}$.

Step 1: Let $\delta>0, j \in \mathrm{N}$ be fixed. Let $\epsilon>0$. Then

case a) $\forall\left(g_{n}\right) \subset K \exists$ subsequence $\left(g_{n_{k}}\right) \exists B_{\epsilon} \subset C_{\delta, j}, w^{*}$-compact, $\nu\left(B_{\epsilon}\right) \geq \nu\left(C_{\delta, j}\right)-\epsilon: \lim \sup _{k, m \rightarrow \infty} \sup _{f \in B_{\epsilon}}\left|f\left(g_{n_{k}}\right)-f\left(g_{n_{m}}\right)\right| \leq \delta$.

case b) $\exists B_{\epsilon} \subset C_{\delta, j}, w^{*}$-compact, $\nu\left(B_{\epsilon}\right) \geq \nu\left(C_{\delta, j}\right)-\epsilon \forall\left(g_{n}\right) \subset K \exists$ subsequence $\left(g_{n_{k}}\right): \lim \sup _{k, m \rightarrow \infty} \sup _{f \in B_{\epsilon}}\left|f\left(g_{n_{k}}\right)-f\left(g_{n_{m}}\right)\right| \leq \delta$.

Proof to step 1: Since $K$ is countable and each $h \in K$ has relatively compact 
range, we may assume that $F$ is separable. Hence, $B_{F^{*}}$ is in the $w^{*}$-topology metrizable and compact. By [7, p. 227] there is a regular Borel-measure $\tau$ on the $w^{*}$-Borel sets, which is measure isomorphic to the Lebesgue measure, thus to the measure $\nu$. Now consider the map $T_{j}: \overline{\operatorname{span}} K \longrightarrow F$, $h \mapsto\|\cdot\|-\lim _{k \rightarrow \infty} h\left(\omega_{k}^{j}\right)$. Then $T_{j}$ is a Rosenthal operator (case a)) resp. an Asplund operator (case b)). Hence, according to [11, p.150-151], we have (consider $T_{j}(h)$ as a measurable function on the compact metric space $B_{F^{*}}$ )

case a) $\forall\left(g_{n}\right) \subset K \exists$ subsequence $\left(g_{n_{k}}\right) \exists \tilde{B}_{\epsilon} \subset B_{F^{*}} w^{*}$-compact, $\tau\left(\tilde{B}_{\epsilon}\right) \geq 1-\epsilon: \lim _{k, m \rightarrow \infty} \sup _{y^{*} \in \tilde{B}_{\epsilon}}\left|\left\langle T_{j}\left(g_{n_{k}}\right)-T_{j}\left(g_{n_{m}}\right), y^{*}\right\rangle\right|=0$.

case b) $\exists \tilde{B}_{\epsilon} \subset B_{F^{*}} w^{*}$-compact, $\quad \tau\left(\tilde{B}_{\epsilon}\right) \geq 1-\epsilon \forall\left(g_{n}\right) \subset K \exists$ subsequence $\left(g_{n_{k}}\right): \lim _{k, m \rightarrow \infty} \sup _{y^{*} \in \tilde{B}_{\epsilon}}\left|\left\langle T_{j}\left(g_{n_{k}}\right)-T_{j}\left(g_{n_{m}}\right), y^{*}\right\rangle\right|=0$.

We define according to Lemma $2.8 B_{\epsilon}:=\left\{f \in \mathscr{C}_{K} ; \exists\left(\omega_{k}\right) \subset \Omega \backslash N \exists y^{*} \in \tilde{B}_{\epsilon}\right.$ such that $\lim _{k \rightarrow \infty} h_{n}\left(\omega_{k}\right)$ exists and $\left.f\left(h_{n}\right)=\lim _{k \rightarrow \infty}\left\langle h_{n}\left(\omega_{k}\right), y^{*}\right\rangle\right\} \cap C_{\delta, j}$. It is easy to verify that $\left\{f \in \mathscr{C}_{K} ; \exists\left(\omega_{k}\right) \subset \Omega \backslash N \exists y^{*} \in \tilde{B}_{\epsilon}\right.$ such that $\lim _{k \rightarrow \infty} h_{n}\left(\omega_{k}\right)$ exists and $\left.f\left(h_{n}\right)=\lim _{k \rightarrow \infty}\left\langle h_{n}\left(\omega_{k}\right), y^{*}\right\rangle\right\}$ is $w^{*}$-compact. Thus $B_{\epsilon}$ is $w^{*}$-compact in $\mathscr{C}_{K}$ and because of the measure isomorphism $\nu\left(B_{\epsilon}\right) \geq \nu\left(C_{\delta, j}\right)-\epsilon$. The definition of $C_{\delta, j}$ and the property of the set $\tilde{B}_{\epsilon}$ imply immediately the assertion of step 1 (note that each $f \in B_{\epsilon}$ has norm distance less or equal than $\delta$ to an $f_{j, y^{*}}, y^{*} \in \tilde{B}_{\epsilon}$ ).

Step 2: Let $\delta>0$ be fixed. Let $\epsilon>0$. Then

case a) $\forall\left(g_{n}\right) \subset K \exists$ subsequence $\left(g_{n_{k}}\right) \exists B_{\epsilon} \subset \mathscr{C}_{K}, w^{*}$-compact, $\nu\left(B_{\epsilon}\right) \geq 1-\epsilon: \lim \sup _{k, m \rightarrow \infty} \sup _{f \in B_{\epsilon}}\left|f\left(g_{n_{k}}\right)-f\left(g_{n_{m}}\right)\right| \leq \delta$.

case b) $\exists B_{\epsilon} \subset \mathscr{C}_{K},, w^{*}$-compact, $\nu\left(B_{\epsilon}\right) \geq 1-\epsilon \forall\left(g_{n}\right) \subset K \exists$ subsequence $\left(g_{n_{k}}\right): \lim \sup _{k, m \rightarrow \infty} \sup _{f \in B_{\epsilon}}\left|f\left(g_{n_{k}}\right)-f\left(g_{n_{m}}\right)\right| \leq \delta$.

Proof to step 2: Since $\mathscr{C}_{K}=\bigcup_{j \in \mathrm{N}} C_{\delta, j}$, there is a $J \in \mathrm{N}$, s.t. $\nu\left(\bigcup_{j=1}^{J} C_{\delta, j}\right) \geq$ $1-\frac{\epsilon}{2}$. For $j=1, \ldots, J$ we apply step 1 and get sets $B_{\frac{\epsilon}{2 j+1}}$ for case a) and b), respectively. Define $B_{\epsilon}:=\bigcap_{j=1}^{J} B_{z_{j+1}}$. Then $\nu\left(B_{\epsilon}\right) \geq 1{ }^{2 /+1} \epsilon$ and $B_{\epsilon}$ fulfills the assertion of step 2 (by going to subsequences, if necessary).

Step 3: Proof of the theorem: Let $\epsilon>0$ be given. We apply step 2 for sequences of $\delta_{l}:=\frac{1}{l}$ and $\left(\frac{\epsilon}{2^{l}}\right)$. Define $\mathscr{C}_{\epsilon}:=\bigcap_{l \in \mathrm{N}} B_{\frac{\epsilon}{2 l}}$. Then we have $\nu\left(\mathscr{C}_{\epsilon}\right) \geq 1-\epsilon$ and (by going to subsequences if necessary):

case a) $\forall\left(g_{n}\right) \subset K \exists$ subsequence $\left(g_{n_{k}}\right) \exists \mathscr{C}_{\epsilon} \subset \mathscr{C}_{K}, w^{*}$-compact, $\nu\left(\mathscr{C}_{\epsilon}\right) \geq 1-\epsilon: \limsup _{k, m \rightarrow \infty} \sup _{f \in \mathscr{C}_{\epsilon}}\left|f\left(g_{n_{k}}\right)-f\left(g_{n_{m}}\right)\right| \leq \delta_{l}=\frac{1}{l}, l \in \mathrm{N}$.

case b) $\exists \mathscr{C}_{\epsilon} \subset \mathscr{C}_{K}, w^{*}$-compact, $\quad \nu\left(\mathscr{C}_{\epsilon}\right) \geq 1-\epsilon \forall\left(g_{n}\right) \subset K \exists$ subsequence $\left(g_{n_{k}}\right): \lim \sup _{k, m \rightarrow \infty} \sup _{f \in \mathscr{C}_{\epsilon}}\left|f\left(g_{n_{k}}\right)-f\left(g_{n_{m}}\right)\right| \leq \delta_{l}=\frac{1}{l}, l \in \mathrm{N}$. 
Since $l \in \mathrm{N}$ is arbitrary, this is the criterion in [15, Thm 3.5,3.6,p.279-280], and the proof is done.

Let us mention that the previous theorem part a) holds for complex Banach spaces as well.

We point out that several of the following results were obtained by different techniques, each of them sometimes easier, but the foregoing theorem is the unique basis for all of them.

COROLlary 2.10. Let $\Omega$ be a compact Hausdorff space. Let $K \subset C(\Omega, F)$ be bounded and countable, such that $\overline{\operatorname{span}}\left\{y^{*} \circ h ; y^{*} \in B_{F^{*}}, h \in K\right\} \subset C(\Omega)$ has separable dual. Then

a) $K$ is conditionally weakly compact, if $\ell_{1} \not \subset F$.

b) $K$ is an Asplund set, provided $F$ is an Asplund space.

Proof. Let $\mu$ be a finite regular Borel measure on $\Omega$, such that $C(\Omega) \hookrightarrow$ $L_{\infty}(\mu)$ isometrically. Then $C(\Omega, F) \hookrightarrow L_{\infty}^{c}(\mu, F)$ isometrically. The assertion follows by the previous theorem.

We apply the previous corollary to the situation of the ideal of compact operators. These two results have earlier been obtained by different methods in [2, Cor. 1.12] resp. [12, Cor. 1.10].

Corollary 2.11. Let E, F be Banach spaces. Then the space of compact operators $\mathscr{K}(E, F)$

a) does not contain $\ell_{1}$, if $E^{*}$ is an Asplund space and $\ell_{1} \not \subset F$.

b) is an Asplund space, if and only if $E^{*}$ and $F$ are Asplund spaces.

Proof. We start with the sufficiency. To show that $\mathscr{K}(E, F)$ does not contain $\ell_{1}$ [resp. is Asplund], it suffices to prove that a bounded and countable set $K \subset \mathscr{K}(E, F)$ is conditionally weakly compact [resp. an Asplund set]. Thus, let $\left(T_{n}\right) \subset \mathscr{K}(E, F)$ be bounded. Since all $T_{n}^{*}$ are compact, we may assume that $E^{*}$ is a separable space which is also an Asplund space. Define in the setting of the previous corollary

$$
\Omega:=B_{E^{* *}} \text { endowed with the } w^{*} \text {-topology, } h_{n}\left(e^{* *}\right):=T_{n}^{* *}\left(e^{* *}\right), n \in \mathrm{N} .
$$

Since the $T_{n}$ are compact, $\left(h_{n}\right) \subset C(\Omega, F)$. Further, we can consider $\left\{y^{*} \circ h_{n} ; y^{*} \in B_{F^{*}}, n \in \mathrm{N}\right\} \subset E^{*} \subset C(\Omega)$. Hence the dual of $\overline{\operatorname{span}}\left\{y^{*} \circ h_{n}\right.$; $\left.y^{*} \in B_{F^{*}}, n \in \mathrm{N}\right\} \subset C(\Omega)$ is separable. Thus the sufficiency is proved in a) resp. b) according to the previous corollary part a) resp. b).

Now assume in b) that $\mathscr{K}(E, F)$ is an Asplund space. Then, since $E^{*}$ and $F$ embed isometrically into $\mathscr{K}(E, F), E^{*}$ and $F$ are Asplund spaces, too.

Another application of Corollary 2.10 is the following 
Corollary 2.12. Let $K_{1} \subset E$ and $K_{2} \subset F$ be bounded Asplund sets. Then $K_{1} \otimes K_{2}:=\left\{x \otimes y: x \in K_{1}, y \in K_{2}\right\}$ is an Asplund set in the injective tensor product $E \check{\otimes} F$.

Proof. Since the Asplund property is determined by countable sets, we may assume that $K_{1}$ and $K_{2}$ are each countable. Hence $E$ may be assumed to be separable. Consider $K_{1}$ as a subset of $C(\Omega)$, where $\Omega$ is the compact metrizable space $B_{E^{*}}$ in the $w^{*}$-topology. Let $K:=K_{1} \otimes K_{2}$. Then $K \subset C(\Omega) \check{\otimes} F=C(\Omega, F) \quad$ and $\quad\left\{y^{*} \circ z ; z \in K, y^{*} \in B_{F^{*}}\right\} \subset \overline{\operatorname{span}} K_{1} \subset C(\Omega)$. Since $K_{1}$ is an Asplund set, $\overline{\operatorname{span}}\left\{y^{*} \circ z ; z \in K, y^{*} \in B_{F^{*}}\right\}$ has a separable dual. Since $K_{2}$ is an Asplund set, $F$ may be assumed to be an Asplund space, so the assertion follows by Corollary 2.10.

The next result is an immediate consequence of Corollary 2.11.

Corollary 2.13. Let $E, F, E_{1}, F_{1}$ be Banach spaces and let $R \in \mathscr{R}(E, F)$ $\left[\right.$ resp. $\left.R \in \mathscr{D}_{\infty}(E, F)\right]$ and $B \in \mathscr{W}\left(E_{1}, F_{1}\right)$. Assume that $E_{1}$ is reflexive, $\ell_{1} \not \subset F$ [resp. $F$ is Asplund] and $S_{R B}\left(\mathscr{L}\left(F_{1}, E\right)\right) \subset \mathscr{K}\left(E_{1}, F\right)$. Then the map $S_{R B}: \mathscr{L}\left(F_{1}, E\right) \rightarrow \mathscr{K}\left(E_{1}, F\right), S_{R B}(T)=R \circ T \circ B$, is a Rosenthal operator [resp. an Asplund operator].

Remark 2.14. If we assume $R \in \mathscr{W}(E, F)$ and $B \in \mathscr{D}_{\infty}\left(E_{1}, F_{1}\right)$ in Cor. 2.13, then the statements do not need to be true. Indeed, let $E=F=R$, $E_{1}=F_{1}=c_{0}$ and $B$ and $R$ be the identity operator. Then $S_{R B}: \ell_{1} \rightarrow \ell_{1}$ is not Rosenthal.

The following corollary is the Main Lemma of Racher [10].

Corollary 2.15. Let $E, F, E_{1}, F_{1}$ be Banach spaces and let $R \in \mathscr{W}(E, F)$ and $B \in \mathscr{W}\left(E_{1}, F_{1}\right)$. Assume that $E_{1}, F$ are reflexive and $R^{* *} \circ \bar{B}_{\mathscr{L}\left(F_{1}, E\right)}^{w^{*}} \circ B$ is contained in $\mathscr{K}\left(E_{1}, F\right)$, where $w^{*}$ is the weak* operator topology of $\mathscr{L}\left(F_{1}, E^{* *}\right)$ induced by the linear functionals $T \mapsto\left\langle y^{*}, T x\right\rangle$ for all $x \in F_{1}, y^{*} \in E^{*}$. Then $S_{R B}: \mathscr{L}\left(F_{1}, E\right) \rightarrow \mathscr{K}\left(E_{1}, F\right), S_{R B}(T)=R \circ T \circ B$, is a weakly compact operator.

Proof. Let $\left(T_{n}\right)$ be a sequence in the closed unit ball $B_{\mathscr{L}\left(F_{1}, E\right)}$ of $\mathscr{L}\left(F_{1}, E\right)$. By Corollary 2.11 a) there is a subsequence which we denote by $\left(T_{n}\right)$ such that for all $x \in E_{1}$ and all $y^{*} \in F^{*}$ the sequence $\left(\left\langle S_{R B}\left(T_{n}\right) x, y^{*}\right\rangle\right)$ is convergent. Since $\mathscr{L}\left(F_{1}, E^{* *}\right) \cong\left(F_{1} \hat{\otimes} E^{*}\right)^{*}$ it follows from Alaoglu's theorem that the sequence $\left(T_{n}\right)$ in $\mathscr{L}\left(F_{1}, E\right)$ has a cluster point $T_{0} \in \mathscr{L}\left(F_{1}, E^{* *}\right)$ in the weak $^{*}$ operator topology. Then $T_{0} \in \bar{B}_{\mathscr{L}\left(F_{1}, E\right)}^{w^{*}}$. Hence $R^{* *} \circ T_{0} \circ B \in$ $\mathscr{K}\left(E_{1}, F\right)$ is a cluster point in the weak operator topology of $\mathscr{L}\left(E_{1}, F\right)$ of the sequence $\left(S_{R B}\left(T_{n}\right)\right) \subset \mathscr{K}\left(E_{1}, F\right)$. Thus the sequence

$$
\left(\left\langle S_{R B}\left(T_{n}\right) x, y^{*}\right\rangle\right) \text { converges to }\left\langle\left(R^{* *} \circ T_{0} \circ B\right) x, y^{*}\right\rangle
$$


for all $x \in E_{1}$ and all $y^{*} \in F^{*}$. Since $R^{* *} \circ T_{n} \circ B$ is compact for all $n \in \mathrm{N} \cup\{0\}$, we may assume that $E_{1}$ is separable. Define $K:=\left\{R^{* *} \circ T_{n} \circ B ; n \in \mathrm{N} \cup\{0\}\right\}$. Since $E_{1}$ is separable $\mathscr{C}_{K}=\left\{f \in(\overline{\operatorname{span}} K)^{*}\right.$; $\exists\left(x_{k}, y_{k}^{*}\right) \in B_{E_{1}}^{\mathrm{N}} \times B_{F^{*}}^{\mathrm{N}}, \lim _{k \rightarrow \infty}\left\langle\left(R^{* *} \circ T_{n} \circ B\right)\left(x_{k}\right), y_{k}^{*}\right\rangle=: f\left(R^{* *} \circ T_{n} \circ B\right)$ exists for all $n \in \mathrm{N} \cup\{0\}\}$. Lemma 2.8 together with [15, p. 275, Prop. 2.5] imply that $\left(S_{R B}\left(T_{n}\right)\right) \in \mathscr{K}\left(E_{1}, F\right)$ converges weakly in $\mathscr{K}\left(E_{1}, F\right)$ to $R^{* *} \circ T_{0} \circ B \in \mathscr{K}\left(E_{1}, F\right)$.

REMARK 2.16. Instead of the last part of the proof of Corollary 2.15 one can use Theorem 2.5 in [2].

\section{Necessary conditions}

In the sequel we consider the converse of the last results in the preceding section, especially Corollary 2.15 . This problem has also been treated by E. Saksman and H. Tylli in [13].

Proposition 3.1. Let $E, F, E_{1}, F_{1}$ be Banach spaces and let $B \in \mathscr{L}\left(E_{1}, F_{1}\right)$ and $R \in \mathscr{L}(E, F)$. Suppose that every non-compact $T \in \mathscr{L}\left(F_{1}, E\right)$ factors through a Banach space with an unconditional basis and that $E$ contains no copy of $c_{0}$. If $S_{R B}: \mathscr{L}\left(F_{1}, E\right) \rightarrow \mathscr{L}\left(E_{1}, F\right), T \mapsto R \circ T \circ B$, is Rosenthal, then $S_{R B}\left(\mathscr{L}\left(F_{1}, E\right)\right) \subset \mathscr{K}\left(E_{1}, F\right)$.

Proof. If not, then there exists a non-compact $T \in \mathscr{L}\left(F_{1}, E\right)$ with $R \circ T \circ B \notin \mathscr{K}\left(E_{1}, F\right)$. Let $T=T_{2} \circ T_{1}$ be factorized through a Banach space $G$ with an unconditional basis $\left(u_{n}\right)$. The unconditional basis constant is denoted by $C$ and $\left(u_{n}^{\prime}\right)$ is the associated sequence of coefficient functionals. Hence there is a sequence of projections $P_{m}: G \rightarrow G$ of finite rank, defined by $P_{m}(x)=\sum_{n=1}^{m} u_{n}^{\prime}(x) u_{n}$. Then $T_{2} \circ P_{m} \circ T_{1} \in \mathscr{K}\left(F_{1}, E\right)$ for every $m \in \mathrm{N}$ and $T_{2} \circ P_{m} \circ T_{1}(x) \rightarrow T(x)$ for every $x \in E$, when $m \rightarrow \infty$. Since $R \circ T \circ B \notin \mathscr{K}\left(E_{1}, F\right)$, the sequence $\left(R \circ T_{2} \circ P_{m} \circ T_{1} \circ B\right)$ is not norm convergent in $\mathscr{K}\left(E_{1}, F\right)$. Hence we can find $\epsilon>0$, a strictly increasing subsequence $\left(m_{k}\right)$ of $(m)$ with $\left\|R \circ A_{k} \circ B\right\|>\epsilon$ for all $k \in \mathrm{N}$, where $A_{k}:=T_{2} \circ P_{m_{2 k}} \circ T_{1}-T_{2} \circ P_{m_{2 k-1}} \circ T_{1}$. Hence $\inf _{k}\left\|A_{k}\right\|>0$. The set $\left\{\sum_{k \in \Delta} A_{k}: \Delta \subset \mathrm{N}\right.$ finite $\}$ is bounded in $\mathscr{K}(E, F)$, since $\left\|\sum_{k \in \Delta} A_{k}\right\| \leq$ $\left\|T_{1}\right\| \cdot\left\|T_{2}\right\| \cdot C$. Therefore, $\sum_{k=1}^{\infty} A_{k}$ is weakly unconditionally Cauchy. By the Bessaga-Pelczynski selection principle we now find a basic subsequence $\left(A_{k_{i}}\right)$ of $\left(A_{k}\right)$. Since $\inf _{i}\left\|A_{k_{i}}\right\|>0$ and $\sum_{i=1}^{\infty} A_{k_{i}}$ is weakly unconditionally Cauchy, the sequence $\left(A_{k_{i}}\right)$ is equivalent to the unit vector basis $\left(e_{i}\right)$ in $c_{0}$. Thus $\mathscr{K}\left(F_{1}, E\right)$ contains a copy of $c_{0}$. Since $E$ contains no copy of $c_{0}$, the proof of Theorem 6, (iii) $\Rightarrow(i i)$, in [6], gives an isomorphic embedding $J: l^{\infty} \rightarrow \mathscr{L}\left(F_{1}, E\right)$ with $J\left(e_{i}\right)=A_{k_{i}}$. For the continuous linear map $S_{R B} \circ J: l^{\infty} \rightarrow \mathscr{L}\left(F_{1}, E\right) \rightarrow \mathscr{L}\left(E_{1}, F\right)$ we have that $\left\|R \circ A_{k_{i}} \circ B\right\|=$ 
$\left\|S_{R B} \circ J\left(e_{i}\right)\right\|>\epsilon$ for all $i \in \mathrm{N}$. Since $S_{R B}$ is Rosenthal it factors through a Banach space $\tilde{G}$ not containing a copy of $\ell_{1}$ with $S_{R B}=U \circ V$. Therefore $\tilde{G}$ does not contain a copy of $\ell_{\infty}$. Now we apply Rosenthal's theorem for nonweakly compact operators on $\ell_{\infty}$ which gives that the continuous linear map $V \circ J: \ell_{\infty} \rightarrow \tilde{G}$ is weakly compact. Hence, by the Orlicz-Pettis' theorem on weak subseries convergence, we get that the series $\sum_{i=1}^{\infty} S_{R B} \circ J\left(e_{i}\right)$ converges in norm in $\mathscr{L}\left(E_{1}, F\right)$. Thus we have a contradiction.

Proposition 3.2. Let $E, F, E_{1}, F_{1}$ be Banach spaces and let $B \in \mathscr{L}\left(E_{1}, F_{1}\right)$ and $R \in \mathscr{L}(E, F)$. Suppose that every non-compact $T \in \mathscr{L}\left(F_{1}, E\right)$ factors through a Banach space with an unconditional basis. If $S_{R B}: \mathscr{K}\left(F_{1}, E\right) \rightarrow$ $\mathscr{K}\left(E_{1}, F\right), \quad T \mapsto R \circ T \circ B, \quad$ is weakly compact, then $S_{R B}\left(\mathscr{L}\left(F_{1}, E\right)\right) \subset$ $\mathscr{K}\left(E_{1}, F\right)$.

Proof. Suppose not, and proceed as in the proof of Proposition 3.1. Then we get an isomorphic embedding $J: c_{0} \rightarrow \mathscr{K}\left(F_{1}, E\right)$ with $J\left(e_{i}\right)=A_{k_{i}}$ and also that $\left\|\left(S_{R B} \circ J\right)\left(e_{i}\right)\right\|>\epsilon>0$ for all $i \in \mathrm{N}$. But, by assumption, the continuous linear map $S_{R B} \circ J: c_{0} \rightarrow \mathscr{K}\left(E_{1}, F\right)$ is compact, so we obtain a contradiction.

Proposition 3.3. Let $E, F, E_{1}, F_{1}$ be Banach spaces and let $B \in \mathscr{L}\left(E_{1}, F_{1}\right)$ and $R \in \mathscr{L}(E, F)$. Suppose that every non-compact $T \in \mathscr{W}\left(F_{1}, E\right)$ has a factorization $T=T_{2} \circ T_{1}$ through a Banach space $G$ with the metric approximation property such that $T_{1} \in \mathscr{W}\left(F_{1}, G\right)$. If $S_{R B}: \mathscr{K}\left(F_{1}, E\right) \rightarrow \mathscr{K}\left(E_{1}, F\right)$, $T \mapsto R \circ T \circ B$, is Dieudonné, then $S_{R B}\left(\mathscr{W}\left(F_{1}, E\right)\right) \subset \mathscr{K}\left(E_{1}, F\right)$.

Proof. We follow D.R. Lewis' idea of proof [8, p. 204]. Let $T=T_{2} \circ T_{1} \in \mathscr{W}\left(F_{1}, E\right)$ be a factorization through a Banach space $G$ with the metric approximation property with $T_{1} \in \mathscr{W}\left(F_{1}, G\right)$. We want to show that $R \circ T \circ B \in \mathscr{K}\left(E_{1}, F\right)$. Let $\left(x_{n}\right) \subset E_{1}$ be a bounded sequence. Put $G_{0}=\overline{\operatorname{span}}\left\{T_{1} \circ B\left(x_{n}\right) ; n \in \mathrm{N}\right\} \subset G$ and $G_{1}=\overline{\operatorname{span}}\left\{T_{1}(y) ;\|y\| \leq 1\right\} \subset G$. Then $G_{1}$ is a WCG Banach space and $G_{0}$ is a separable, closed linear subspace of $G_{1}$. By a theorem of Amir and Lindenstrauss on separable-valued projections in WCG-spaces (see [5, Theorem 3, p. 149]), there exists a separable, closed linear subspace $H$ of $G_{1}$ containing $G_{0}$ and a projection $P: G_{1} \rightarrow H$ with $\|P\|=1$. Now, let $\left(y_{i}\right)$ be a norm dense sequence in $H$. Since $G$ has the metric approximation property, there is a sequence $\left(U_{n}\right) \in \mathscr{F}(G, G)$ of norm one operators such that $\left\|y-U_{n}(y)\right\|<\frac{1}{n}\|y\|$ for all $y \in \operatorname{span}\left\{y_{1}, \ldots, y_{n}\right\}$. For each $n \in \mathrm{N}$, define $V_{n}:=T_{2} \circ U_{n} \circ P \circ T_{1} \in \mathscr{K}\left(F_{1}, E\right)$. Now it follows as in [8, p.204] that $V_{n}^{* *} z^{* *} \rightarrow\left(T_{2} \circ P \circ T_{1}^{* *}\right) z^{* *}$ in norm in $E$ for every $z^{* *} \in F_{1}^{* *}$. Then it is not hard to show that $\left(V_{n}\right)$ is weakly Cauchy in $\mathscr{K}\left(F_{1}, E\right)$. By assumption, $S_{R B}\left(V_{n}\right) \rightarrow V \in \mathscr{K}\left(E_{1}, F\right)$ weakly in $\mathscr{K}\left(E_{1}, F\right)$. For every $x \in E_{1}$, $S_{R B}\left(V_{n}\right) x \rightarrow V x$ weakly in $F$ and $\left(R \circ V_{n} \circ B\right) x \rightarrow\left(R \circ T_{2} \circ P \circ T_{1} \circ B\right) x$ in 
norm in $F$. Thus $V x=\left(R \circ T_{2} \circ P \circ T_{1} \circ B\right) x$ for every $x \in E_{1}$. Hence, for every $n, V\left(x_{n}\right)=(R \circ T \circ B)\left(x_{n}\right)$, from which follows that $R \circ T \circ B \in$ $\mathscr{K}\left(E_{1}, F\right)$.

The equivalence between $c$ ) and $d$ ) in our next corollary has been obtained by Saksman and Tylli in [13].

COROllary 3.4. Let $E$ be a reflexive Banach space with the approximation property and let $B \in \mathscr{L}(E, E)$ and $R \in \mathscr{L}(E, E)$. Then the following statements are equivalent:

a) $S_{R B}: \mathscr{K}(E, E) \rightarrow \mathscr{K}(E, E)$ is weakly compact,

b) $S_{R B}: \mathscr{K}(E, E) \rightarrow \mathscr{K}(E, E)$ is Dieudonné,

c) $S_{R B}(\mathscr{L}(E, E)) \subset \mathscr{K}(E, E)$,

d) $S_{R B}: \mathscr{L}(E, E) \rightarrow \mathscr{L}(E, E)$ is weakly compact.

Proof. By Corollary 2.15 resp. Proposition 3.3 we have that c) implies d) resp. b) implies c). The other implications are obvious.

Our results concerning the converse of Corollary 2.15 can be stated as follows.

Theorem 3.5. Let $E, F, E_{1}, F_{1}$ be Banach spaces and let $B \in \mathscr{L}\left(E_{1}, F_{1}\right)$ and $R \in \mathscr{L}(E, F)$ be non-zero operators. Suppose that every non-compact $T \in \mathscr{L}\left(F_{1}, E\right)$ factors through a Banach space with an unconditional basis or has a factorization $T=T_{2} \circ T_{1}$ through a Banach space $G$ with the metric approximation property such that $T_{1} \in \mathscr{W}\left(F_{1}, G\right)$. If $S_{R B}: \mathscr{L}\left(F_{1}, E\right) \rightarrow$ $\mathscr{L}\left(E_{1}, F\right), T \mapsto R \circ T \circ B$, is weakly compact, then $R^{* *} \circ \bar{B}_{\mathscr{L}\left(F_{1}, E\right)}^{w^{*}} \circ B \subset$ $\mathscr{K}\left(E_{1}, F\right)$, where $w^{*}$ is the weak ${ }^{*}$ operator topology of $\mathscr{L}\left(F_{1}, E^{* *}\right)$.

Proof. By Proposition 3.2 resp. Proposition 3.3 we have that $S_{R B}\left(\mathscr{L}\left(F_{1}, E\right)\right) \subset \mathscr{K}\left(E_{1}, F\right)$.

Now let $A \in \bar{B}_{\mathscr{L}\left(F_{1}, E\right)}^{w^{*}} \subset \mathscr{L}\left(F_{1}, E^{* *}\right)$. Then there is a net $\left(T_{\alpha}\right) \in B_{\mathscr{L}\left(F_{1}, E\right)}$ which converges to $A$ in the weak* operator topology. The assumption and Proposition 2.1 imply that $R \in \mathscr{W}(E, F)$. Thus the net $\left(R \circ T_{\alpha} \circ B\right) \subset$ $\mathscr{K}\left(E_{1}, F\right)$ converges to $R^{* *} \circ A \circ B \in \mathscr{L}\left(E_{1}, F\right)$ in the weak operator topology. Since $\left(S_{R B}\left(T_{\alpha}\right)\right)$ is relatively weakly compact in $\mathscr{K}\left(E_{1}, F\right)$, at least a subnet converges weakly to an operator $T_{0}$, which is compact, since $\mathscr{K}\left(E_{1}, F\right)$ is weakly closed in $\mathscr{L}\left(E_{1}, F\right)$. Since the weak operator topology is coarser than the weak topology, we conclude that $R^{* *} \circ A \circ B=$ $T_{0} \in \mathscr{K}\left(E_{1}, F\right)$. 


\section{REFERENCES}

1. R. D. Bourgin, Geometric aspects of convex sets with the Radon-Nikodym property, Lecture Notes in Math. 993 (1983).

2. H. S. Collins and W. Ruess, Weak compactness in spaces of compact operators and vectorvalued functions, Pacific J. Math. 106, (1983), 45-71.

3. J. Diestel and B. Faires, Remarks on the classical Banach operator ideals, Proc. Amer. Math. Soc. 58, (1976), 189-196.

4. A. Defant and K. Floret, Tensor Norms and Operator Ideals, North Holland (1993).

5. J. Diestel, Geometry of Banach Spaces, Lect. Notes in Math. 483 (1975).

6. N. Kalton, Spaces of compact operators, Math. Ann. 208 (1973), 267-278.

7. C. Kuratowski, Topologie I, Monografje Matematyczne Vol. 3, Warsawa (1933).

8. D. R. Lewis, Conditional weak compactness in certain inductive tensor products, Math. Ann. 201 (1971), 201-209.

9. A. Pietsch, Operator Ideals, North Holland (1980).

10. G. Racher, On the tensor product of weakly compact operators, Math. Ann. 294 (1992), 267275.

11. L. H. Riddle and J. J. Uhl, Martingales and the fine line between Asplund spaces and spaces not containing a copy of $\ell_{1}$ in: Martingale Theory in Harmonic Analysis and Banach Spaces, Lecture Notes in Math. 939 (1983) 145-156.

12. W. Ruess and C. Stegall, Extreme points in duals of operator spaces, Math. Ann. 261 (1982), $535-546$.

13. E. Saksman and H. O. Tylli, Weak compactness of multiplication operators on spaces of bounded linear operators, Math. Scand. 70 (1992), 91-111.

14. E. Saksman and H. O. Tylli, Weak essential spectra of multiplication operators on spaces of bounded linear operators, Math. Ann. 299 (1994), 299-309.

15. G. Schlüchtermann, Weak Cauchy in $L_{\infty}(\mu, X)$, Studia Math. 116 (3) (1995), 271-281.

16. C. Stegall, The duality between Asplund spaces and spaces with the Radon-Nikodym property, Israel J. Math. 29 (1978), 408-412.

17. K. Vala, On compact sets of compact operators, Ann. Acad. Sci. Fenn. Ser. A I. Math 351 (1964). 